\title{
A Coherent Multiple Beamforming Network for a Non-uniform Circular Antenna Array
}

\author{
Armando ARCE ${ }^{1,2}$, Enrique STEVENS-NAVARRO ${ }^{2}$, Marco CARDENAS-JUAREZ ${ }^{2}$, \\ Ulises PINEDA-RICO ${ }^{2}$, David H. COVARRUBIAS ${ }^{3}$ \\ ${ }^{1}$ Catedras CONACYT, Universidad Autonoma de San Luis Potosi, 78290 San Luis Potosi, Mexico \\ ${ }^{2}$ Faculty of Sciences, Universidad Autonoma de San Luis Potosi, 78290 San Luis Potosi, Mexico \\ ${ }^{3}$ Dept. of Electronics and Telecommunications, CICESE Research Centre, 22860 Ensenada, Mexico
}

armando.arce@uaslp.mx, \{estevens, mcardenas, u_pineda $\} @$ fc.uaslp.mx, dacoro@ cicese.mx

Submitted May 29, 2018 / Accepted January 10, 2019

\begin{abstract}
This work proposes and describes a modular and innovative beamforming network (BFN) to feed a nonequally spaced circular antenna array. The structure is based on a set of alternated power combiners and dividers that delivers a Gaussian-like amplitude distribution and coherent (in-phase) signals. A multiple beam antenna system to generate two main beams in the same aperture with a coherent network for a nonuniform array with beam shaping and beam steering properties is simulated and analyzed. Furthermore, a comparative analysis based on uniform and nonuniform circular antenna arrays fed by the proposed coherent network is conducted. The complex signals and the nonuniform circular aperture are optimized using the well known differential evolution technique. Numerical experiments show the efficiency and improvement of the coherent network with a nonuniform aperture over uniform, with an advantage in average equal to $1.8 \mathrm{~dB}$ of directivity and $-2 \mathrm{~dB}$ of side lobe level. Moreover, the simulation results exhibit an aperture reuse and complexity reduction of the proposed coherent network configuration compared with a conventional antenna array with direct feeding, where each main beam is shaped and steered with the half of control signal inputs.
\end{abstract}

\section{Keywords}

Multiple beamforming network, non-uniform circular array, differential evolution, coherent beamforming network, multiple beams

\section{Introduction}

The technology of multiple-beam antennas exhibits desirable capabilities in modern antenna applications, where its antenna array based solution offers flexibility on the radiation operation. Generally, these systems can radiate multiple independent countoured beams with advanced features as beam steering, high-directive beam, among others intel- ligent beam operations. Such radiation features in general require a beamforming network to properly provide a predefined phase and amplitudes distribution to the array radiating elements [1], [2]. These multiple beamforming networks are constituted by multiple input ports and output ports that are connected to the antenna array. The most notable and studied work based on lossless multiple beamforming networks lies in Blass [3], Butler [4] and Nolen [5] matrices. Despite, these lossless matrices have low-loss properties, the orthogonal behavior at the output tend to a more restrictive pattern shape, inhibiting the flexibility in the overall design [6].

In this context, coherent networks represent an alternative solution that improves flexibility with beam shaping capability and enhanced beam steering, working with coherent (in-phase) output distribution related to complex inputs controls. Recently, a coherent network known as coherently radiating periodic structure beamforming network was introduced with a simpler topology based on just power combiners and dividers [7]. The coherent beamforming network delivers a coherent Gaussian-like amplitude tapered excitation law. In addition, this versatile multiple beamforming network have been studied with diverse approaches since its introduction, such as an efficiency analysis of the structure based on the original concept, design and simplification of the coherent network in different antenna array geometries and physical implementations based on enhancements of the feeding network and its basic components [8-10]. Although the authors have been addressed the nonuniform linear array fed by a coherent beamforming network in [11], to the best of our knowledge the nonuniform aperture on circular arrays has not been previously analyzed in this feeding network on literature.

This paper presents as main contribution a detailed analysis of the performance of an antenna system that exploits the nonuniformity in circular arrays with a design of a coherent beamforming network. Thus, in this work is proposed a feeding network configuration based on coherently radiating periodic structures to feed a nonuniformly spaced 
circular array. The required complex excitations at the input ports and the interlement spacing are obtained by the differential evolution method. To demonstrate the performance of a nonuniformly spaced circular array a comparative analysis with a uniform circular array in a two-beam system is simulated.

The remainder of the paper is organized as follows. Section 2 address theorically the model of a typical coherently radiating periodic structure as feeding network, the nonuniform circular array formulation as a part of the antenna system and the optimization method applied. Section 3 presents the experimental setup for the coherent multibeam configuration and its optimizer, in the same section simulation results for the antenna system are performed and analyzed. Finally, in Sec. 4 conclusions of this work are presented.

\section{Problem Formulation}

\subsection{Beamforming Network Theoretical Model}

In this work, the proposed feeding network is based on the concept of coherently radiating periodic structures beamforming network (CORPS-BFN or for simplicity C-BFN) where power dividers and combiners, employed as basic nodes, are alternated and used to propagate the power until the antenna elements [7]. In this arrangement, a complete feeding network topology can be implemented connecting and alternating basic nodes through successive layers, as is illustrated in Fig. 1. A layer is composed by an arrangement of basic nodes, power combiners nodes (C) in each layer are connected to power dividers (D) in a subsequent layer. At the ouput, the network delivers a Gaussian-like amplitude and in-phase excitation law at each input port (true time delay) [12].

The flexibility of the network based on a basic node, makes possible to establish different configurations with a varying number of inputs, outputs, and layers for a specific antenna system design. Furthermore, the intrinsic characteristics of this coherent network allows to control the beamshaping and beam steering, even in multi-beam designs. Typical C-BFN designs consider a greater number of output ports $(N)$ than input ports $(M)$ where the number of layers are given by $N-M$. The 3-port component employed as basic node and used for power division and combination is represented by the following scattering parameters, and can be written as [7]:

$$
[\mathbf{S}]_{\text {node }}=\left[\begin{array}{ccc}
0 & \mathrm{j} / \sqrt{2} & \mathrm{j} / \sqrt{2} \\
\mathrm{j} / \sqrt{2} & 0 & 0 \\
\mathrm{j} / \sqrt{2} & 0 & 0
\end{array}\right] .
$$

The ideal scattering matrix ensures no interaction between input signals assuming matching conditions at all ports.
To evaluate the output of a C-BFN, we can use a transfer matrix per layer $\left[\mathbf{T}_{m}\right]$ in transmit mode given by the following expression [8]:

$$
\left[\boldsymbol{\alpha}_{m+1}\right]_{(m+1,1)}=\left[\mathbf{T}_{m}\right]_{(m+1, m)} \cdot\left[\boldsymbol{\alpha}_{m}\right]_{(m, 1)},
$$

in which $\left[\boldsymbol{\alpha}_{m+1}\right]$ denotes the output vector and $\left[\boldsymbol{\alpha}_{m}\right]$ is the input vector (complex excitation) at the feeding ports, both in the evaluated layer. The output vector is introduced as input vector in the next layer. In (2), $m$ denotes the input ports and subscripts define the size of the matrices. Considering a C-BFN topology with more than one layer, with $N$ output ports and $M$ input ports, the behavior of the output excitation law $\left[\alpha_{N}\right]$ of the feeding network can be found using (2) successively, and is expressed as:

$$
\left[\boldsymbol{\alpha}_{N}\right]_{(N, 1)}=\left(\prod_{m=N-1}^{M}\left[\mathbf{T}_{m}\right]\right) \cdot\left[\boldsymbol{\alpha}_{M}\right]_{(M, 1)}
$$

where $\left[\mathbf{T}_{m}\right]$ represents the transfer matrices of each layer in the feeding network and $m \in[N-1, N-2, \ldots, M]$. The transfer matrices can be properly defined based on the transfer coefficients of the basic node in (1). From the matrix it can be inferred that the columns denote power division and rows represent power combination. A general transfer matrix of C-BFN can be formulated as:

$$
\left[\mathbf{T}_{m}\right]=\frac{1}{2}\left[\begin{array}{ccccc}
\sqrt{2} & 0 & 0 & \cdots & 0 \\
1 & 1 & 0 & \cdots & 0 \\
0 & 1 & 1 & & \vdots \\
0 & 0 & \ddots & \ddots & 0 \\
\vdots & \vdots & & 1 & 1 \\
0 & 0 & \cdots & 0 & \sqrt{2}
\end{array}\right]_{(N, M)}
$$

For multi-beam configurations, a change for a numeric value of 1 on the coefficients located at $(1,1)$ and $(m+1, m)$ is suggested due to edge losses that only affect limited values in the matrix. In any case, is feasible to simulate and analyze a C-BFN, using a topology based on Fig. 1 and assessing (2), (3) and (4). Thus, the coherent behavior of signals throughout the structure can be simulated in a programming language (e.g. Python, $\mathrm{C}$, among others) or numerical software (e.g., MATLAB, Octave, etc.). In the same context, there are studies focused on physical implementations of C-BFN that support its mathematical theory, this research is based on replicate the basic constituting component or unit cell, by means of different multi-port devices such as Gysel power dividers/combiners [7], circular in-phase hybrid rings for wideband purposes [12] or modified multi-port Gysel devices for isolation improvements [13].

Next, the mathematical expressions for the array factor for a nonuniformly spaced circular antenna array considered as a part of the antenna system are described. 


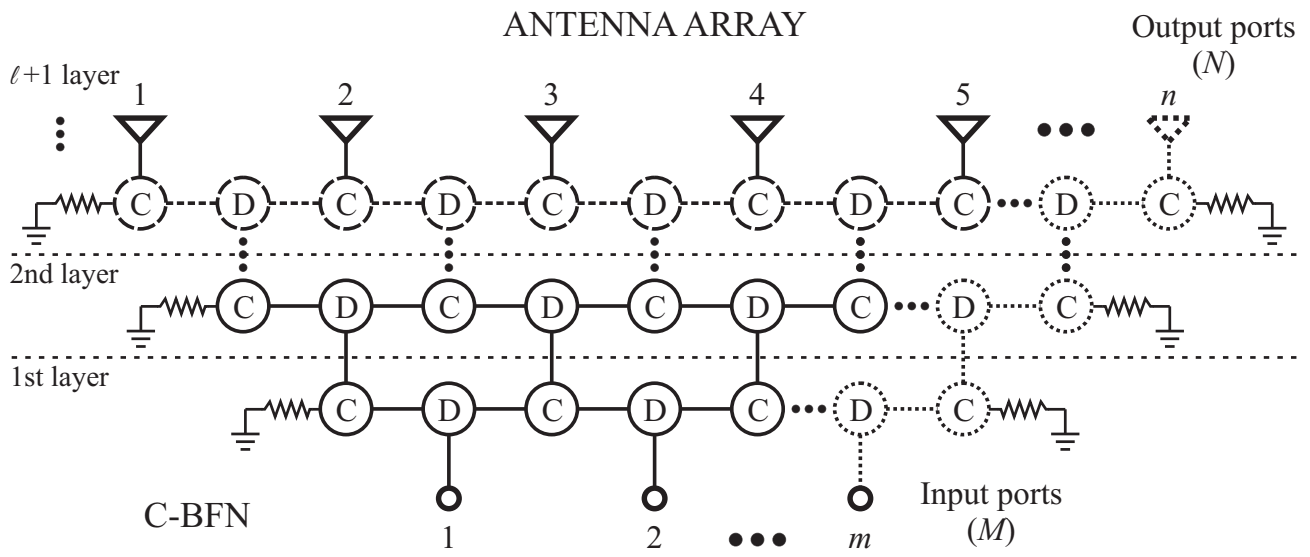

Fig. 1. Schematic of a typical C-BFN topology based on basic nodes for power combination/division.

\subsection{Non-uniform Circular Antenna Array}

Let us now consider a circular array geometry composed of $\mathrm{N}$ isotropic sources along a circle of radius $r$ in a $x-y$ plane. Its array factor $(A F(\phi))$ for the bidimensional plane $x-y(\theta=90)$ can be written as [14]:

$$
A F(\phi)=I_{n} \exp \left[\mathrm{j}\left(k r \cos \left(\phi-\phi_{n}\right)+\beta_{n}\right)\right]
$$

where $\beta_{n}=-k r\left(\cos \phi_{0}-\phi_{n}\right)$ denotes the excitation phase of the $n$-th element, used to direct the peak of the main lobe in $\phi_{0}$ direction. Considering this circular geometry of antenna elements in connection with the BFN, the array factor in function of a set of complex inputs $\mathbf{I}$ with nonuniform antenna element locations $\mathbf{d}$ to be optimized can be rewritten from (5) as:

$$
A F(\phi, \mathbf{I}, \mathbf{d})=I_{n} \exp \left[\mathrm{j} k r\left(\cos \left(\phi-\phi_{n}\right)-\cos \left(\phi_{0}-\phi_{n}\right)\right)\right]
$$

where $k=2 \pi / \lambda, \lambda$ is the operating wavelength, $\phi$ is the azimuthal angle, $I_{n}$ denotes the complex weights $\left(A_{n}=\mathrm{e}^{\mathrm{j} \xi_{n}}\right)$ at the $n$-th input port of the feeding network, the radius of the array is $r=N d \lambda / 2 \pi$ and the angular location of the $n$-th element along the feeding network is $\phi_{n}=2 \pi(n-1) / N$ for $n=1,2, \ldots, N$.

In this work, the beam pattern synthesis in the antenna system is done by an stochastic algorithm which handles several combinations of possible solutions to find a nearoptimal solution for the non equally spaced circular antenna array [15-17] and the feeding system that meets specific objectives. Furthermore, due to the degrees of freedom involved and the complexity in antenna synthesis optimization, a metaheuristic based on evolutionary computation is used and described in the next section.

\subsection{Optimization Process}

Differential Evolution (DE) is the stochastic real parameter optimization technique used in the antenna feed system to obtain a set of solutions of antenna element locations and complex excitations. DE is an efficient and reliable evolutionary algorithm classified as a population-based optimizer successfully used on electromagnetics problems including beam pattern synthesis and antenna design [18-20]. In this work, DE algorithm is used just as an optimization tool for its proven efficiency on synthesis problems, a comparative analysis between this algorithm and other population based algorithms (e.g. Particle Swarm Optimization (PSO), Genetic Algorithms (GA), among others) is out of the scope of this paper. Furthermore, this optimization technique (DE) exhibits a better performance in comparison with other traditional population based optimizers, based on literature and our previous research experience. DE is much more simple and straightforward to implement, the number of control parameters in DE are few and the space complexity of DE can be considered low. Despite, another population based algorithms can be used to optimize the antenna system with similar good performance in terms of side lobe level and directivity, the DE technique was used in this paper based on the time of convergence and the advantages mentioned previously.

In DE algorithm the real-valued parameter vectors to be optimized forms a candidate solution in a multidimensional problem. DE begins with these randomly initiated vectors (or agents) forming a population $\left(N_{\mathrm{p}}\right)$ where some of these solutions are selected and geometrically perturbed with a scaled difference of two randomly chosen population vectors. The lead role in the optimization is performed by the mutation scheme, where for each d-dimensional real-valued vector $\varepsilon_{i}^{G}=\left[\varepsilon_{i, 1}^{G}, \varepsilon_{i, 2}^{G}, \ldots, \varepsilon_{i, d}^{G}\right]$ of the population at the $G$-th iteration, the mutation or donor vector $\left(\mathbf{v}_{i}^{G+1}\right)$ is given by:

$$
\mathbf{v}_{d}^{G+1}=\varepsilon_{\mathrm{r} 1}^{G}+F\left(\varepsilon_{\mathrm{r} 2}^{G}-\varepsilon_{\mathrm{r} 3}^{G}\right)
$$

in which $F$ is a positive control parameter for scaling the difference vectors $\left(\varepsilon_{\mathrm{r} 2}^{G}-\varepsilon_{\mathrm{r} 3}^{G}\right)$ which controls the length of the exploration, known as mutation factor or differential weight (typically with a range between $[0.4,1]$ ). The indices $r_{1}, r_{2}$ and $r_{3}$ are mutually exclusive integers randomly chosen from the population $N_{\mathrm{p}}$. Please refer to [21], [22], for a more detailed information of this evolutionary algorithm. 
The process applied in this work to find sets of valid solutions is presented below: DE begins randomly generating vectors (or agents) as candidate solutions. These proposed solutions are grouped by a vector of real numbers representing element-to-element arc spacings $\mathbf{d}=\left[d_{1}, d_{2}, \ldots, d_{n}\right]$ and a vector of complex numbers denoting amplitudes and phases $\mathbf{I}=\left[A_{1} \mathrm{e}^{\mathrm{j} \xi_{1}}, A_{2} \mathrm{e}^{\mathrm{j} \xi_{2}}, \ldots, A_{M} \mathrm{e}^{\mathrm{j} \xi_{M}}\right]$. By this way, each agent in the population proposes a radiation pattern that meets with certain characteristics of side lobe level and directivity for a predefined direction of interest in the antenna system. Subsequently, the mutation operator (7) in the algorithm expands the search space, followed by the crossover operator which promotes the diversity of the population and controls the number of components inherited from mutation with a certain probability crossover rate (CR), affecting convergence speed. Thereupon the selection operator warrants the survival of the fittest solutions in subsequents generations and reduces the possibility of stagnation in DE population. At the end of the algorithm, we obtain a global solution that generates a multibeam pattern in the same aperture under the design characteristics imposed, i.e., a beam pattern with maximum directivity and minimum side lobe level in a predefined direction for each beam in our case study. To achieve the above predefined design characteristics, the related synthesis problem can be formulated mathematically as a constrained optimization as follows:

$$
\begin{array}{ll}
\min _{\mathbf{I}, \mathbf{d}} & \left\{\frac{\left|A F\left(\phi_{\mathrm{SL}}, \mathbf{I}, \mathbf{d}\right)\right|}{\left|A F\left(\phi_{\max }, \mathbf{I}, \mathbf{d}\right)\right|}\right\}+\left\{\frac{1}{D(\phi, \mathbf{I}, \mathbf{d})}\right\} \\
\text { s.t. } & \left|A F\left(\phi_{\max }, \mathbf{I}, \mathbf{d}\right)\right|=1, \\
& \mathbf{I}=\left\{A_{m} \mathrm{e}^{\mathrm{j} \xi_{m}} \mid \mathbf{I} \in \mathbb{I}\right\}, \forall m \in M, \\
& \mathbf{d}=\left\{d_{n} \mid \mathbf{d} \in \mathbb{D}\right\}, \forall n \in N .
\end{array}
$$

In (8), $\phi_{\mathrm{SL}}$ is the angle where maximum side lobe is attained on either side of main lobe, $\phi_{\max }$ is the main beam direction, $\mathbb{D}$ is the antenna interelement location domain, and I represents the amplitude and phase domain. The optimization task can be expressed as to find the near-optimal solution of inter-element spacing matrix $\mathbf{d}$ for the nonuniform circular array and the complex excitations matrix I for the BFN's input ports, in order to get reduced side lobes and high directivity in a specific direction of interest.

\section{Numerical Experiments}

In this section, the simulation setup is described and the numerical experiments are carried out in MATLAB to assess and validate the effectiveness of a $\mathrm{C}$-BFN with a nonuniform circular array (NUCA), for simplicity a two-beam system is proposed and analyzed.The objective is to assess the two-beam radiation pattern conformed by the complete antenna system, i.e., the antenna array and the feeding network. Moreover, the results are compared with the same feeding network but with a uniform circular array (UCA) to contrast performance.

\subsection{Experimental Setup}

The DE algorithm was implemented for the assessment of the array factor generated in the azimuth plane $\left(0^{\circ} \leq \phi \leq 360^{\circ}\right)$ in the cut of the $\theta=90^{\circ}$. The nonuniform antenna array with circular geometry is composed by 30 antenna elements with random nonuniform element-toelement arc spacing between $d_{\min }=0.25 \lambda$ to $d_{\max }=\lambda$ and $d=0.5 \lambda$ for the uniform case. The DE strategy with random selection, one difference vector, and binomial crossover is applied to the antenna system, with the following parameters: the algorithm initializes with a population $\left(N_{\mathrm{p}}\right)$ of 500 individuals with the same maximum number of iterations ensuring a good sampling of the solution space, a mutation factor set in $F=0.5$ with a crossover rate (CR) of 0.9 . The stochastic algorithm was executed for 20 independent trials for each direction of interest in each beam.

The antenna system is shown in Fig. 2, the schematic shows a circular array composed by 30 non equally spaced antenna elements with a feeding system based on 29 feeding ports implemented in a C-BFN of one layer. This coherent network allows to control two independent beams simultaneously by alternating the feeding ports $(M)$ in the beamforming network, as shown in the bottom of Fig. 2 . The indices at the input ports indicate the number of beam or signal applied, i.e., each index number belongs to a group of complex inputs to conform one of the two beams. In this configuration, the first beam is shaped and controlled by 15 intercalated input ports that delivers its signal throughout the aperture (i.e., 30 output ports directly connected to the same number of antennas). Similarly, the 14 remaining intercalated input ports are excited to manipulate 28 antenna elements where the total output conforms the second orthogonal beam sharing the same antenna array elements, except for the elements at the ends. The output ports $(N)$ of the beamforming network are directly connected to the antenna elements in the circular geometry.

\subsection{Simulation Results}

The normalized power patterns of the two-beam antenna system fed by the coherent beamforming network with nonuniform radiant elements, are shown in Fig. 3. In this example case, the main beam was directed in three different directions of interest for each beam, where the maximum radiation is scanned along the azimuth plane and set in $20^{\circ}, 140^{\circ}$ and $260^{\circ}$ for beam number 1 and aimed at $70^{\circ}, 190^{\circ}$ and $310^{\circ}$ for beam number 2. Moreover, by the particular type of geometry on the proposed antenna system and by the optimization task realized by $\mathrm{DE}$, it is possible to obtain as can be observed in Fig. 3 an isolation level and directivity that remain almost constant along the azimuthal plane.

The optimized power patterns in this multibeam design demonstrate the capacity of the antenna system to handle the trade-off between side lobe level (SLL) and directivity (D) in different predefined directions for each of the main beams. 


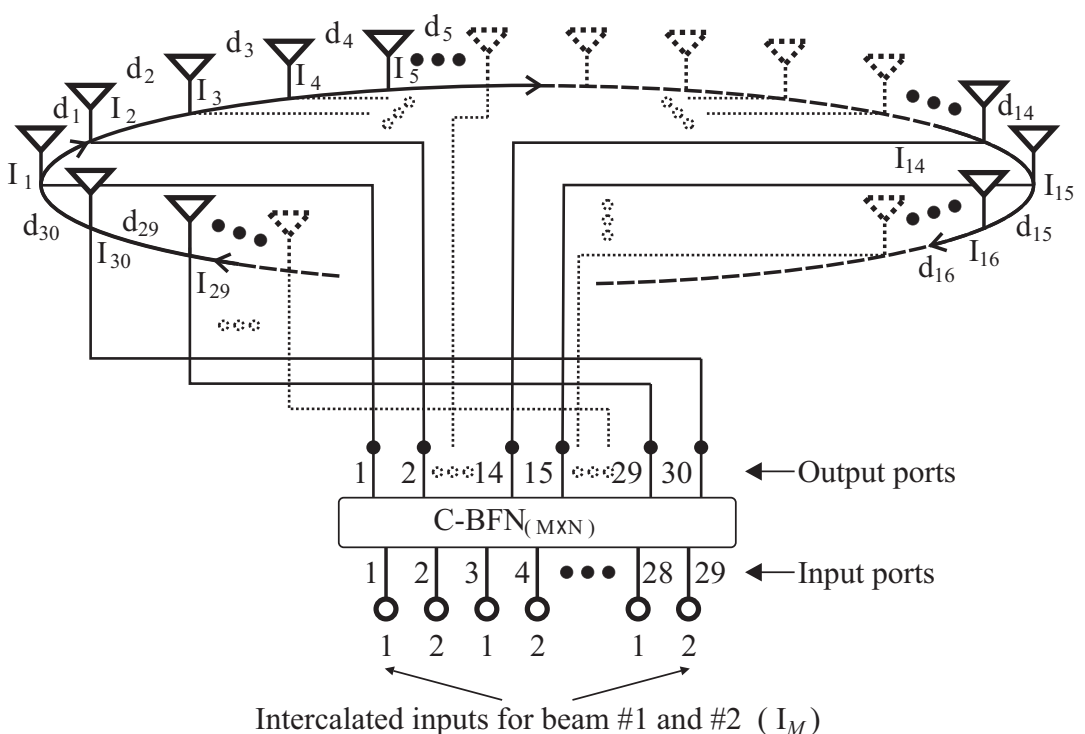

Fig. 2. Schematic of a two-beam antenna system based on a C-BFN for a nonuniformly spaced circular array.

Despite the optimization is uniformly weighted in the cost function, a high directivity is obtained by the coherent network. The optimized values for the antenna elements distribution and the complex excitations used at the BFN's input ports for the example case, are shown in Tab. 1. The table details the numerical solution to generate each beam pattern illustrated in Fig. 3, aimed to different azimuthal angles.

In this particular case study of a two-beam design, the C-BFN can conform and steer each beam to any direction of interest under the window of visibility, by feeding $N-1$ input ports alternately and exploiting the nonuniformity between elements. The feeding network that uses alternated subsets of complex inputs for each beam, employs 15 input ports for properly feed 30 antenna elements to control the beam number 1 and it uses the remaining 14 input ports for exciting 28 antenna elements for beam number 2 . In this design, the structure allows to reuse radiators between orthogonal beams thanks to a working principle of operation of a C-BFN called coherent coupling. In this way, a C-BFN of one layer topology, conform and scan two beams sharing the same aperture and reducing the feeding ports, representing an advantage against typical phased antenna array with direct feeding.

A comparative analysis of a C-BFN that feeds a circular array antenna array is performed. In this evaluation, the antenna system shown in Fig. 2 is considered with the uniform and nonuniform interelement spacing variants for the circular geometry. On each configuration, the array factor generated is analyzed and the antenna system is previously optimized by $\mathrm{DE}$ algorithm with the simulation parameters described in Sec. 3.1 in both designs. To test the possible improvements of a non equallly spaced over equally spaced circular arrays in a coherent beamforming network with multi-beam capabilities, the worst and best solutions obtained of a NUCA were contrasted with best result of a UCA with the same C-BFN configuration under equal simulation settings and several trials for a comprehensive comparative evaluation.
Examples of the array factor's behavior conformed by the proposed two-beam design of a C-BFN for uniform and nonuniform interelement spaced circular array are illustrated in Figs. 4 and 5. Figure 4 shows the main beam set in $\phi_{0}=140^{\circ}$ and generated by the feeding ports dedicated to conform beam number 1 , for both circular configurations i.e., nonuniform and uniform cases. Specifically, in this example, the main lobe is generated by 15 input ports exciting all the aperture i.e., 30 antenna elements, in all cases. The numerical values of SLL and D are $-11.3 \mathrm{~dB}$ and $14 \mathrm{~dB}$, for the best solution of a $\mathrm{C}-\mathrm{BFN}$ in a uniform circular array (UCA + CBFN (best case)), $-11.7 \mathrm{~dB}$ and $15.5 \mathrm{~dB}$ in the worst instance of a $\mathrm{C}-\mathrm{BFN}$ with a nonuniform aperture (NUCA + CBFN (worst case)), and $-13.5 \mathrm{~dB}$ and $16 \mathrm{~dB}$ for the best result of a $\mathrm{C}-\mathrm{BFN}$ in a nonuniform interelement spacing (NUCA + CBFN (best case)).

Similarly, Fig. 5 demonstrates the performance of beam number 2 directed at $\phi_{0}=190^{\circ}$ for all the cases. In this figure, the best normalized pattern of a UCA + CBFN achieves $-12 \mathrm{~dB}$ and $14.1 \mathrm{~dB}$ of SLL and $\mathrm{D}$, respectively, in contrast the worst array factor of a NUCA + CBFN shows $-12.1 \mathrm{~dB}$ and $15.1 \mathrm{~dB}$, and $-14 \mathrm{~dB}$ and $15.7 \mathrm{~dB}$ for the best normalized pattern of a NUCA + CBFN. In this example case, the main beam is conformed by 14 input ports exciting 28 output ports. Despite the fact, that beam number 2 is generated by a smaller antenna aperture, it obtains a similar performance in terms of SLL and D with respect beam number 1. In this specific configuration, the output ports on the ends of the feeding network are not excited, due to the confined propagation of energy inside the structure between power dividers/combiners. However, this shortcoming does not represent a significant performance disadvantage for beam number 2 .

Furthermore, in Figs. 4 and 5 the general performance of the worst case of a normalized pattern in a NUCA with a C-BFN is slightly better than the best case of a radiation pattern in a UCA with the same feeding network, 


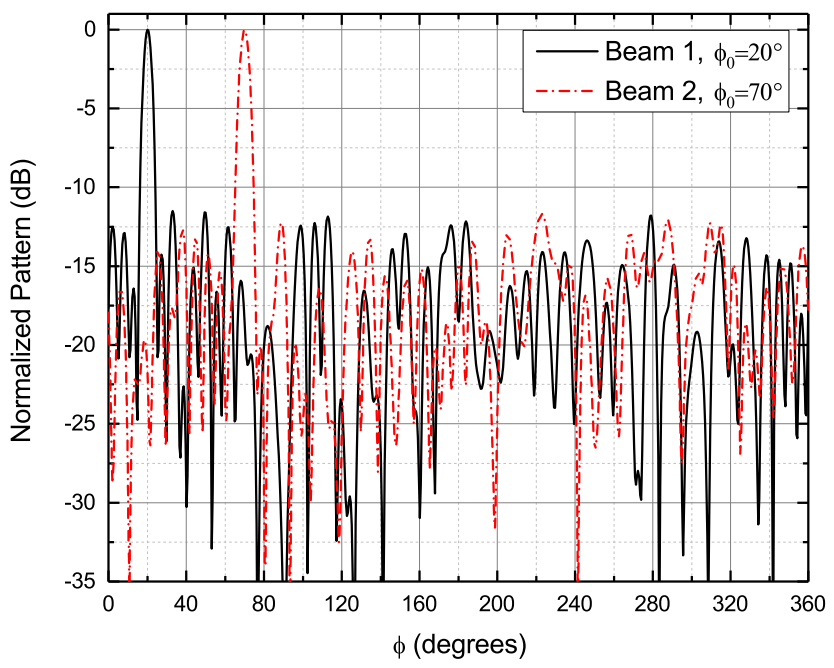

(a) Beam 1 is directed at $20^{\circ}$ and beam 2 at $70^{\circ}$

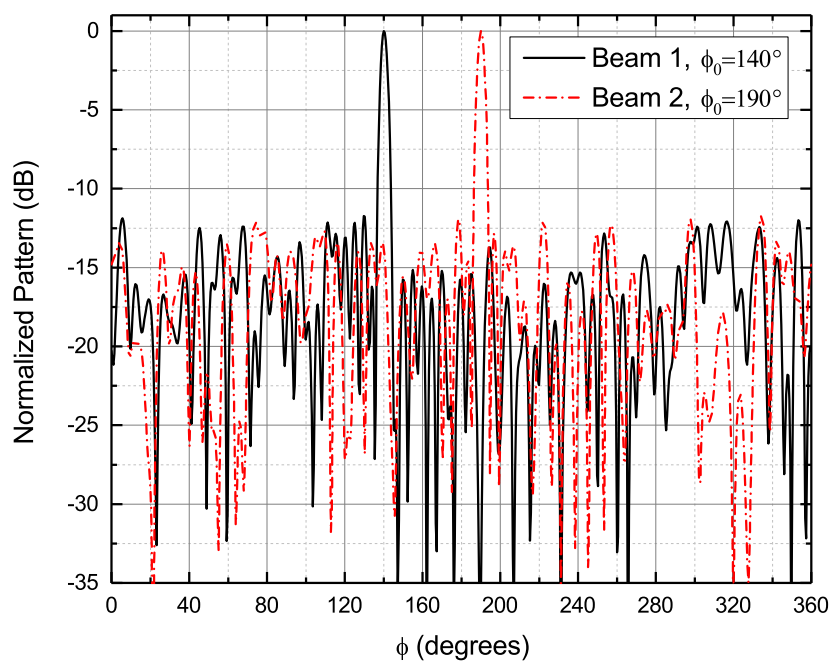

(b) Beam 1 is directed at $140^{\circ}$ and beam 2 at $190^{\circ}$.

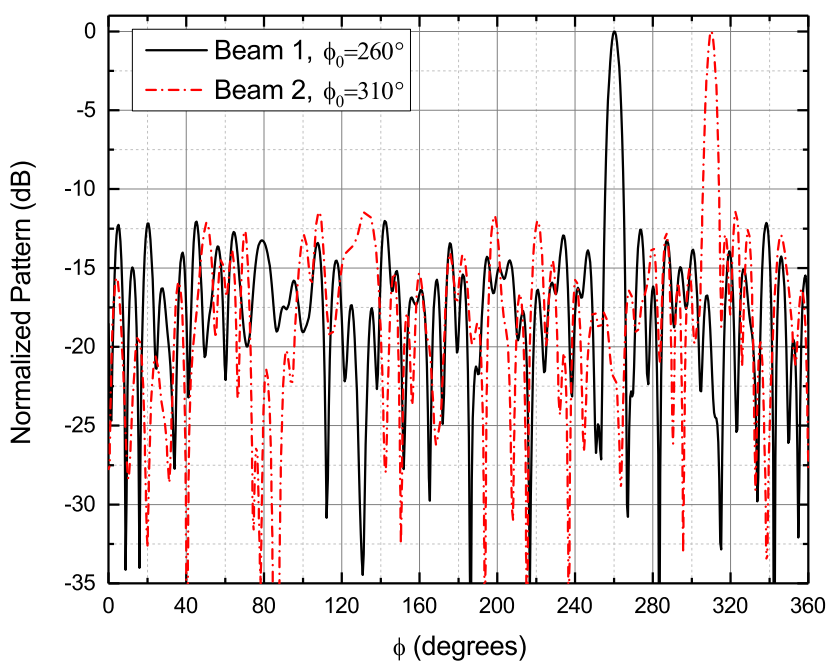

(c) Beam 1 is directed at $260^{\circ}$ and beam 2 at $310^{\circ}$.

Fig. 3. Normalized patterns generated by the two-beam feeding network with a nonuniform circular array optimized by differential evolution. even over all the visibility window as shown in Tab. 2. This behavior demonstrate that a coherent beamforming network based on nonuniformly spaced circular array outperforms in equal conditions a uniformly spaced circular array fed by the same feeding network, in terms of side lobe level and directivity. Moreover, both figures prove a considerable superiority in the two-beam antenna system performance of the best case of NUCA with a C-BFN over the best case of a UCA with this coherent network, the advantage in average is equal to $-2 \mathrm{~dB}$ of side lobe level and $1.8 \mathrm{~dB}$ of directivity in both beams in all the steering range, as can be observed in Tab. 2 .

Specific numerical values of side lobe level and directivity for the two-beam design with the proposed configuration of coherent network, for the uniform and nonuniform cases are presented in Tab. 2. Table 2 collects information of the best numerical values of SLL and D obtained by the uniform circular array and the worst and best values of the aforementioned parameters generated by the nonuniformly spaced circular array. A C-BFN feeding a circular antenna array in the uniform and nonuniform circular geometries preserves basic properties of circular arrays, with almost constant numerical values of side lobe level and directivity throughout the steering range. Specifically, the proposed configuration of CBFN to feed the circular array based on subsets of alternated inputs to conform one of the two beams tends to high numerical values of directivity over side lobe level on fair and equal conditions on the optimization process. From the comparative analysis related to circular arrays shown in Figs. 4, 5, and Tab. 2, the experiments ensure that a C-BFN with a nonuniform circular array has better performance than a uniform circular array in terms of directivity and side lobe level.

Finally, to extend the analysis of the proposed antenna system, simulations with more than two beams were carried out. In this case, the antenna system with a circular nonuniform aperture and the coherent beamforming network shown previously in Fig. 2 was used. In this array based on 30 non equispaced radiators and with 29 feeding ports, multiple independent beams were shaped and controlled by alternating the feeding ports (M). A multiple beam example controlling 5 beams simultaneously by the coherent network is shown in Fig. 6. In this specific case, the main lobes were located in five different predefined directions and set in $45^{\circ}, 115^{\circ}, 185^{\circ}, 255^{\circ}$ and $325^{\circ}$. The optimized power patterns in this multiple beam design prove the performance of the antenna system to handle more than two beams simultaneously, while maintaining the trade-off between minimum side lobe level and maximum directivity.

Table 3 shows the mean values of SLL and D of a main lobe located at 45 degrees (taken as reference) for more than two beams on the aperture. In this numerical experiment, the whole aperture is shared with 3, 4 and 5 beams simultaneously demonstrating the capacity of the network to conform and control more than two beams. 


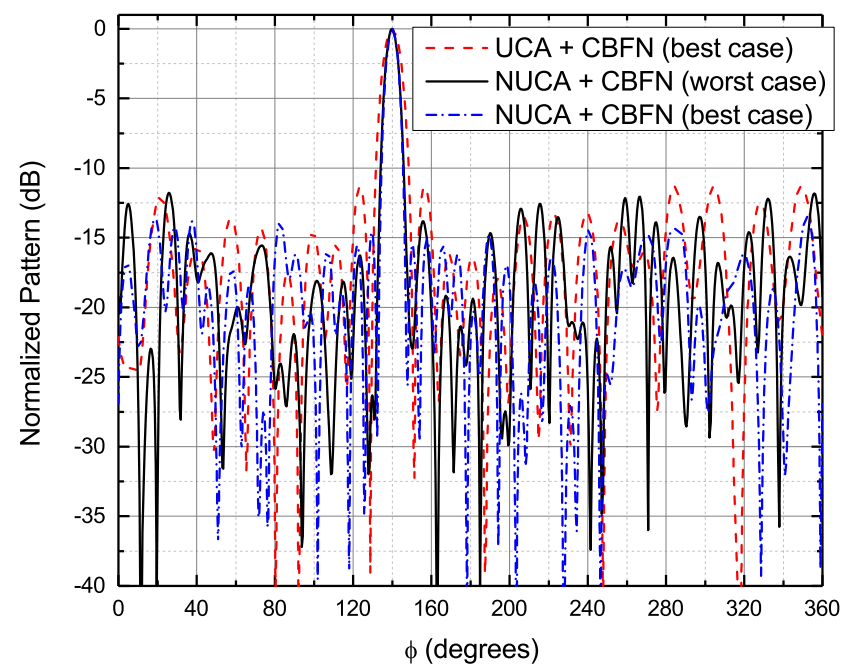

Fig. 4. Comparison of radiation patterns for UCA and NUCA generated by the proposed multi-beam C-BFN, illustrating an example of beam number 1 steered at $140^{\circ}$.

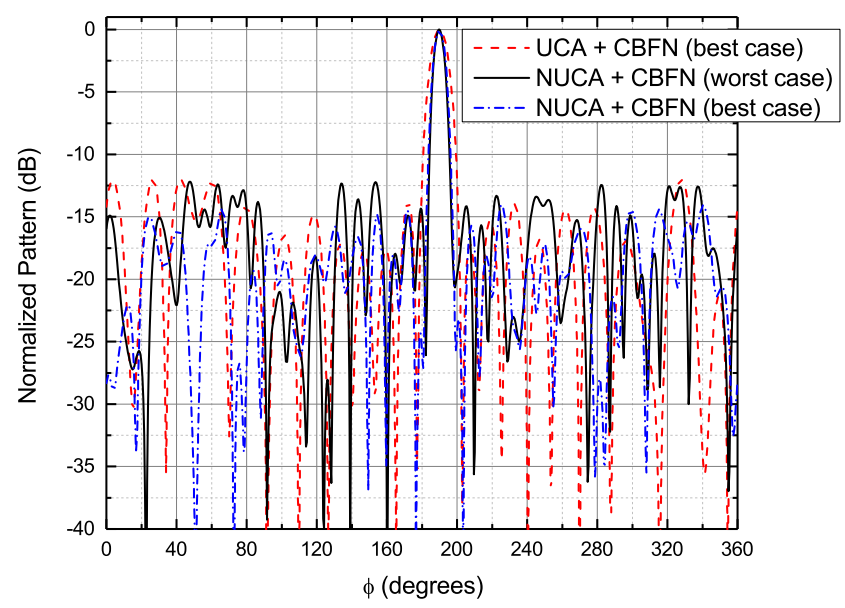

Fig. 5. Comparison of radiation patterns for UCA and NUCA generated by the proposed multi-beam C-BFN, illustrating an example of beam number 2 steered at $190^{\circ}$.

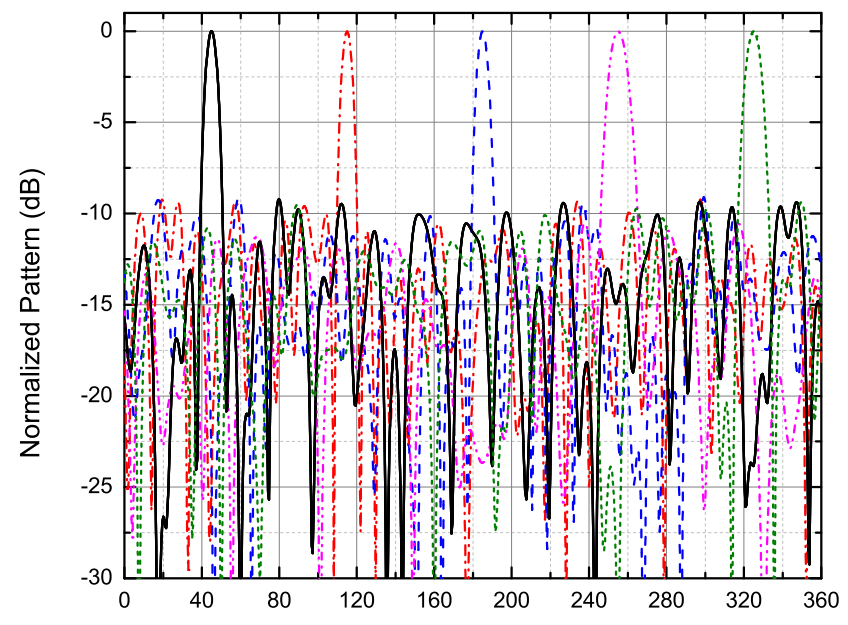

Fig. 6. A multibeam example with 5 beams generated simultaneously sharing the antenna aperture.
Furthermore, the experiment exposes the clear trade-off between the number of beams on the aperture and the antenna parameters under study (SLL and D). In this case, the SLL and $\mathrm{D}$ parameters decreases as the number of independent beams increases on the aperture. For example, in the specific case of 5 main beams the SLL and D mean values are $-9.1 \mathrm{~dB}$ and $13.3 \mathrm{~dB}$ with a marked difference in comparison with 3 beams of approximately $3 \mathrm{~dB}$ and $1.3 \mathrm{~dB}$ of SLL and $\mathrm{D}$, respectively. Note that in a 3 beam configuration, each beam is conformed by 10 intercalated input ports that excite and share 20 antenna elements. In contrast, in a 5 beam configuration, each beam is shaped with 6 intercalated inputs that feed and share 12 radiators on the aperture.

The proposed feeding network design based on C-BFN for a circular antenna arrays, exploits its intrinsic working principles to improve features over a conventional circular aperture in a phased array with direct feeding, such as sharing the antenna elements coherently for multibeam applications and the reduction of control signal inputs. Moreover, the superiority of a nonuniform circular in addition with a C-BFN is clearly demonstrated overcoming the uniform aperture with the same coherent network. Despite that in some cases the above affirmation could be conjectured specially in antenna arrays with direct feeding, in this work is demostrated with an specific configuration using a coherent feeding network based on numeric experimentation. For the two-beam design, each main beam is steered and shaped with $M=N / 2$, i.e., the half of feeding ports are excited to control the double of output ports. Similarly, numeric simulations with more than two beams demonstrate an equivalent behavior where the main lobe for each beam is controlled with $M=N / B$, where $B$ denotes the total number of beams on the antenna aperture. Thus, each intercalated input signal shares the feeding ports exciting and controlling in general the double of radiators per beam. Thus, an increase of the total number of beams on the antenna aperture, decrease the effective aperture for each beam affecting directly and negatively the antenna parameters (SLL and D in this specific case). The above findings on the numerical experimentation, define the limit of the proposed antenna system configuration (Fig. 2), i.e., the maximum directivity and minimum isolation level achieved on a nonuniform circular aperture with feeding network under the simulation conditions exposed previously. Despite a twobeam antenna system is proposed as a main study case, it was proved that the configuration based on intercalated inputs can handle more than two beams on the same antenna aperture. However, this particular configuration is not unique, different C-BFN configurations could be implemented by incrementing the layers and varying or grouping input/output ports for the beams required. A further analysis of nonuniform apertures with different antenna geometries could improve the overall performance, e.g., aperiodic concentric ring arrays. Due the complexity reduction, multibeam capabilities and the shaping performance over all the steering range in a nonuniform circular aperture using an approach based on C-BFN, this network is a useful candidate for various multibeam applications over conventional direct feeding. 


\begin{tabular}{|c|c|c|c|c|c|c|c|c|}
\hline \multicolumn{3}{|c|}{ Beam 1: $20^{\circ}$, Beam 2: $70^{\circ}$} & \multicolumn{3}{|c|}{ Beam 1: $140^{\circ}$, Beam 2: $190^{\circ}$} & \multicolumn{3}{|c|}{ Beam 1: $260^{\circ}$, Beam 2: $310^{\circ}$} \\
\hline$A_{m}$ & $\mathbf{e}^{\mathbf{j} \xi_{m}}[\operatorname{deg}]$ & $\boldsymbol{d}_{\boldsymbol{n}}[\lambda]$ & $A_{m}$ & $\mathrm{e}^{\mathbf{j} \xi_{m}}[\mathrm{deg}]$ & $\boldsymbol{d}_{\boldsymbol{n}}[\lambda]$ & $A_{m}$ & $\mathrm{e}^{\mathbf{j} \xi_{m}}[\mathrm{deg}]$ & $\boldsymbol{d}_{\boldsymbol{n}}[\lambda]$ \\
\hline 0.6422 & -61.4 & 0.696 & 0.5033 & 82.1 & 0.578 & 0.4881 & -31 & 0.505 \\
\hline 0.6743 & -143.4 & 0.769 & 0.5020 & 28.2 & 0.748 & 0.6549 & 176.6 & 0.749 \\
\hline 0.8213 & 90.9 & 0.456 & 0.6679 & -121.5 & 0.752 & 0.8751 & 30.8 & 0.908 \\
\hline 0.4455 & 162.4 & 0.761 & 0.5379 & 26.4 & 0.446 & 0.5994 & -146.3 & 0.516 \\
\hline 0.9238 & -86.7 & 0.503 & 0.1449 & 71.4 & 0.333 & 0.4370 & -27.1 & 0.592 \\
\hline 0.7729 & 128.2 & 0.567 & 0.7691 & 38.1 & 0.443 & 0.4496 & -172.1 & 0.647 \\
\hline 0.4695 & 97.3 & 0.557 & 0.5787 & 90.5 & 0.344 & 0.9083 & 34.6 & 0.448 \\
\hline 0.3967 & 119.3 & 0.940 & 0.5428 & 53 & 0.928 & 0.5336 & -170.8 & 0.615 \\
\hline 0.5325 & 85.4 & 0.538 & 0.3375 & -76 & 0.780 & 0.9825 & 25.2 & 0.786 \\
\hline 0.5133 & 127.5 & 0.470 & 0.6098 & 22.3 & 0.524 & 0.9186 & -174.2 & 0.485 \\
\hline 0.5750 & -72.3 & 0.770 & 0.8441 & 112.8 & 0.367 & 0.3728 & 11.6 & 0.816 \\
\hline 0.5681 & -129.9 & 0.877 & 0.8326 & 24.4 & 0.675 & 0.7918 & 155.5 & 0.320 \\
\hline 0.5542 & -95.3 & 0.451 & 0.4590 & -96.6 & 0.370 & 0.5783 & -35.7 & 0.954 \\
\hline 0.3357 & 83.6 & 0.825 & 0.9220 & 36.3 & 0.546 & 0.6273 & 141.9 & 0.404 \\
\hline 0.7513 & 90.5 & 0.504 & 0.4599 & 110.6 & 0.899 & 0.2989 & -36.9 & 0.714 \\
\hline 0.4248 & 137 & 0.709 & 0.7517 & 39 & 0.715 & 0.5171 & 125.4 & 0.840 \\
\hline 0.5526 & -68.3 & 0.621 & 0.5680 & -139.1 & 0.558 & 0.9285 & 32.6 & 0.289 \\
\hline 0.6586 & 117.6 & 0.566 & 0.6659 & 41.8 & 0.999 & 0.4723 & 175.2 & 0.758 \\
\hline 0.5942 & 78.8 & 0.738 & 0.8859 & 117.4 & 0.627 & 0.9778 & -29.4 & 0.415 \\
\hline 0.6000 & -121.7 & 0.725 & 0.9034 & 27.9 & 0.380 & 0.3022 & 137 & 0.672 \\
\hline 0.7775 & 81.3 & 0.624 & 0.3123 & 101.8 & 0.539 & 0.7215 & 29.3 & 0.705 \\
\hline 0.4447 & -118.3 & 0.563 & 0.6941 & 12.5 & 0.637 & 0.5584 & -160.3 & 0.599 \\
\hline 0.5554 & -75.4 & 0.661 & 0.9453 & 117.7 & 0.315 & 0.1744 & 39.2 & 0.482 \\
\hline 0.8329 & 91.7 & 0.599 & 0.1524 & 63.1 & 0.532 & 0.2743 & -140 & 0.654 \\
\hline 0.2653 & -46.1 & 0.507 & 0.8399 & -99.4 & 0.446 & 0.7606 & -53.9 & 0.784 \\
\hline 0.6234 & 111.3 & 0.437 & 0.6027 & 36.3 & 0.635 & 0.9064 & -141.3 & 0.749 \\
\hline 0.4584 & -89.4 & 0.470 & 0.5845 & 109.6 & 0.663 & 0.3865 & -56.7 & 0.414 \\
\hline 0.5948 & 143 & 0.342 & 0.9460 & 14.4 & 0.445 & 0.5682 & -147.1 & 0.732 \\
\hline 0.9663 & -96.3 & 0.644 & 0.2008 & 107.7 & 0.593 & 0.2842 & 23.1 & 0.480 \\
\hline- & - & 0.852 & - & - & 0.333 & - & - & 0.562 \\
\hline
\end{tabular}

Tab. 1. Complex excitation and interelement space distributions for the two-beam design at different directions of interest shown in Fig. 3.

\begin{tabular}{cccccccc}
\hline $\begin{array}{c}\text { Number } \\
\text { of beam }\end{array}$ & $\begin{array}{c}\text { Main beam direction } \\
\boldsymbol{\phi}_{\mathbf{0}}[\mathrm{deg}]\end{array}$ & \multicolumn{2}{c}{ UCA + C-BFN (best case) } & \multicolumn{2}{c}{ NUCA + C-BFN (worst case) } & \multicolumn{2}{c}{ NUCA + C-BFN (best case) } \\
SLL $[\mathrm{dB}]$ & $\mathbf{D}[\mathrm{dB}]$ & SLL [dB] & D [dB] & SLL [dB] & D [dB] \\
\hline \multirow{2}{*}{1} & 20 & -11.9 & 14 & -12.2 & 15.2 & -13.7 & 15.9 \\
& 140 & -11.3 & 14 & -11.7 & 15.5 & -13.5 & 16 \\
\hline & 260 & -11.4 & 13.9 & -11.5 & 15.3 & -14 & 15.8 \\
\hline \multirow{2}{*}{2} & 70 & -11.6 & 13.8 & -11.7 & 15.6 & -13.6 & 15.7 \\
& 190 & -12 & 14.1 & -12.1 & 15.1 & -14 & 15.7 \\
& 310 & -11.2 & 14 & -11.4 & 15.3 & -13.3 & 16 \\
\hline
\end{tabular}

Tab. 2. Numerical values of side lobe level and directivity for the two-beam antenna system with uniform and nonuniform circular array.

\begin{tabular}{ccc}
\hline $\begin{array}{c}\text { Number of beams } \\
\text { on the aperture }\end{array}$ & \multicolumn{2}{c}{ Main beam directed at $\mathbf{4 5}^{\circ}$} \\
\hline 3 & -11.9 & 14.6 \\
4 & -9.9 & 14.1 \\
5 & -9.1 & 13.3 \\
\hline
\end{tabular}

Tab. 3. Mean values of side lobe level and directivity with a reference main lobe located at $45^{\circ}$ for different number of beams on the aperture. 


\section{Conclusions}

The design of a coherent beamforming network for a two-beam antenna system in a nonuniformly spaced circular array with shaping and steering capabilities has been presented. In this work, the behavior of normalized power patterns generated by the proposed feeding network in a uniform and nonuniform circular aperture were compared and analyzed. The numerical experiments demonstrated that the proposed coherent beamforming network configuration conforms multiple independent beams with scanning properties in the same antenna aperture, taking advantage of the nonuniformity of the antenna array with a reduced complexity of the antenna system. Specifically, presented results of the proposed coherent network configuration based on alternated feeding ports in a nonuniform circular aperture shows intrinsic high directivity values $(D \geq 15 \mathrm{~dB})$ with a considerable reduction of control signals inputs to control each beam $(N / B=M)$ compared with a typical direct feeding system $(N=M)$. The nonuniform aperture improves its uniform counterpart by at least $2 \mathrm{~dB}$ in average approximately, in terms of side lobe level and directivity parameters. Moreover, the coherent beamforming network configuration exhibits the advantages and flexibility of using these coherent networks in nonuniformly spaced circular arrays for multibeam applications with scanning capabilities.

\section{Acknowledgments}

This work is supported the Mexican National Council of Science and Technology (CONACYT) under the project Catedras (No. 872).

\section{References}

[1] BLAKE, J., NYGREN, E., SCHENNUM, G. Beamforming networks for spacecraft antennas. In Proceedings of 1984 Antennas and Propagation Society International Symposium. Boston (USA), 1984, vol. 22, p. 158-161. DOI: 10.1109/APS.1984.1149264

[2] ANGELETTI, P., LISI, M. Multimode beamforming networks for space applications. IEEE Antennas and Propagation Magazine, 2014, vol. 56, no. 1, p. 62-78. ISSN: 1045-9243. DOI: 10.1109/MAP.2014.6821760

[3] BLASS, J. Multidirectional antenna - A new approach to stacked beams. In Proceedings of 1958 IRE International Convention Record. New York (USA), 1958, vol. 8, p. 48-50. DOI: 10.1109/IRECON.1960.1150892

[4] BUTLER, J., LOWE, R. Beam-forming matrix simplifies design of electronically scanned antennas. Electronic Design, 1961, vol. 9, p. $170-173$.

[5] NOLEN, J. Synthesis of multiple beam networks for arbitrary illuminations. Ph.D. Dissertation. Baltimore (USA): Radio Division, Bendix Corp., 1965.

[6] ALLEN, J. A theoretical limitation on the formation of lossless multiple beams in linear arrays. IRE Transactions on Antennas and Propagation, 1961, vol. 9, no. 4, p. 350-352. ISSN: 0096-1973. DOI: 10.1109/TAP.1961.1145014
[7] BETANCOURT, D., DEL RIO BOCIO, C. A Novel Methodology to Feed Phased Array Antennas. IEEE Transactions on Antennas and Propagation, 2007, vol. 55, no. 9, p. 2489-2494. ISSN: 0018-926X. DOI: 10.1109/TAP.2007.904133

[8] FERRANDO, N., FONSECA, N. J. G. Investigations on the efficiency of array fed coherently radiating periodic structure beam forming networks. IEEE Transactions on Antennas and Propagation, 2011, vol. 59, no. 2, p. 493-502. ISSN: 0018-926X. DOI: 10.1109/TAP.2010.2096392

[9] ARCE, A., PANDURO, M. A., COVARRUBIAS, D. H., et al. An approach for simplifying a multiple beam-forming network for concentric ring arrays using CORPS. Journal of Electromagnetic Waves and Applications, 2014, vol. 28, no. 4, p. 430-441. ISSN: 0920-5071. DOI: $10.1080 / 09205071.2013 .870685$

[10] ARCE, A., YEPES, L. F., COVARRUBIAS, D. H., et al. A new approach in the simplification of a multiple-beam forming network based on CORPS using compressive arrays. International Journal of Antennas and Propagation, 2012, vol. 2012, no. 251865, p. 8. DOI: $10.1155 / 2012 / 251865$

[11] ARCE, A., CARDENAS-JUAREZ, M., PINEDA-RICO, U., et al. A multiple beamforming network for unequally spaced linear array based on CORPS. International Journal of Antennas and Propagation, 2014, vol. 2015, no. 757989, p. 7. DOI: 10.1155/2015/757989

[12] FONSECA, N. J. G. Design and implementation of a closed cylindrical BFN-fed circular array antenna for multiple-beam coverage in Azimuth. IEEE Transactions on Antennas and Propagation, 2012, vol. 60, no. 2, p. 863-869. ISSN: 0018-926X. DOI: 10.1109/TAP.2011.2174956

[13] ZAKER, R., ABDIPOUR, A., TAVAKOLI, A. Full-wave simulation, design and implementation of a new combination of antenna array feed network integrated in low profile microstrip technology. Analog Integrated Circuits and Signal Processing, 2014, vol. 80, no. 3, p. 507-517. ISSN: 1573-1979. DOI: 10.1007/s10470-014-0325-X

[14] BALANIS, C. A. Antenna Theory: Analysis and Design. 3rd ed., rev. New York (USA): John Wiley \& Sons, 2005. ISBN: 047166782X

[15] ENACHE, F., DEPARATEANU, D., POPESCU, F. Optimal design of circular antenna array using genetic algorithms. In Proceedings of the 9th International Conference on Electronics, Computers and Artificial Intelligence (ECAI). Targoviste (Romania), 2017, p. 1-6. DOI: 10.1109/ECAI.2017.8166392

[16] ZHAO, X., YANG, Q., ZHANG, Y. Design of non-uniform circular antenna arrays by convex optimization. In Proceedings of the 10th European Conference on Antennas and Propagation (EuCAP). Davos (Switzerland), 2016, p. 1-4. DOI: 10.1109/EuCAP.2016.7481628

[17] CHAKRAVARTHY VEDULA, V. S. S. S., CHOWDARY PALADUGA, S. R., RAO PRITHVI, M. Synthesis of Circular Array Antenna for Sidelobe Level and Aperture Size Control Using Flower Pollination Algorithm. International Journal of Antennas and Propagation, 2015, vol. 2015, no. 819712, 9 p. ISSN: 2210-6502. DOI: $10.1155 / 2015 / 819712$

[18] DEB, A., ROY, J. S., GUPTA, B. A differential evolution performance comparison: Comparing how various differential evolution algorithms perform in designing microstrip antennas and arrays. IEEE Antennas and Propagation Magazine, 2018, vol. 60, no. 1, p. 51-61. ISSN: 1045-9243. DOI: 10.1109/MAP.2017.2774146

[19] GOUDOS, S. Antenna design using binary differential evolution: Application to discrete-valued design problems. IEEE Antennas and Propagation Magazine, 2017, vol. 59, no. 1, p. 74-93. ISSN: 1045-9243. DOI: 10.1109/MAP.2016.2630041

[20] YANG, H., YANG, S., NI, D., NIE, Z. Pattern synthesis approach for circularly polarised four-dimensional antenna arrays. IET Microwaves, Antennas Propagation, 2015, vol. 9, no. 10, p. 1004-1008. ISSN: 1751-8725. DOI: 10.1049/iet-map.2014.0544 
[21] DAS, S., MULLICK, S. S., SUGANTHAN, P. N. Recent advances in differential evolution - An updated survey. Swarm and Evolutionary Computation, 2016, vol. 27, p. 1-30. ISSN: 2210-6502. DOI: $10.1016 /$ j.swevo.2016.01.004

[22] ROCCA, P., OLIVERI, G., MASSA, A. Pattern synthesis approach for circularly polarised four-dimensional antenna arrays. IET Microwaves, Antennas Propagation, 2015, vol. 9, no. 10, p. 1004-1008. ISSN: 1751-8725. DOI: 10.1049/iet-map.2014.0544

\section{About the Authors ...}

Armando ARCE received the Ph.D. degree in Telecommunications and Electronic Engineering from CICESE Research Centre in 2012. Currently, he is a Catedras CONACYT Researcher at Faculty of Sciences of the UASLP, Mexico. His research interests are in beamforming networks and antenna analysis and synthesis. He is member of the IEEE Antennas and Propagation Society since 2015 and member of the Mexican National System of Researchers since 2013.

Enrique STEVENS-NAVARRO received the Ph.D. degree in Electrical and Computer Engineering from the University of British Columbia, Canada in 2008. Currently, he is an Associate Professor at Faculty of Sciences of the UASLP, Mexico. His research interests are in wireless communications and networking. He is member of the IEEE Communications Society since 1999, member of the IEICE Communications Society since 2010 and member of the Mexican National System of Researchers since 2010. Dr. Stevens-Navarro is Senior Member of the IEEE since 2015 and regular member of the Mexican Academy of Sciences since 2017.
Marco CARDENAS-JUAREZ received the Ph.D. degree in Signal Processing for Communications from the University of Leeds, United Kingdom in 2012. Currently, he is an Associate Professor at Faculty of Sciences of the UASLP, Mexico. His research interests are in signal processing and communications. He is member of the IEEE Communications and Signal Processing societies since 2010, and member of the Mexican National System of Researchers since 2015. Dr. Cardenas-Juarez is Senior Member of the IEEE since 2016.

Ulises PINEDA-RICO received the Ph.D. degree in Electrical and Electronic Engineering from The University of Manchester, United Kingdom in 2009. Currently, he is an Associate Professor at Faculty of Sciences of the UASLP, Mexico. His main research interests are MIMO wireless technologies, unmanned aerial vehicles (UAV) and related. Dr. Pineda-Rico is member of the IEICE and IEEE Communications Societies and member of the Mexican National System of Researchers since 2010.

David H. COVARRUBIAS received his Ph.D. degree in Telecommunications from the Polytechnic University of Catalonia, Barcelona, Spain in 1999. He served as a Research Fellow at the Centre for Scientific Research and Higher Education of Ensenada (CICESE), Baja California, Mexico. He has investigated cellular mobile communications, providing state of the art in the areas of synthesis array antennas, source localization algorithms, and space-time precoders. He currently works at the Technology Platform 5G Wireless Communications that regulates the development and regulation of the next cellular mobile communications generation. 